\title{
An unusual cause of recurrent pneumonia
}

\author{
Neil A McAndrew, Gethin R Ellis, G Jeffrey Green
}

A 57-year-old man was admitted with right lower lobe pneumonia. He had had rheumatic fever as a child and 21 years later had a mitral valve replacement. He developed prosthetic valve endocarditis and the valve was replaced 9 months later. Para-prosthetic valve regurgitation developed and 4 years later he again required mitral valve replacement. He had been successfully treated for radiologically proven right lower lobe pneumonia several months after the second valve replacement but did not have a significant history of pulmonary disease. Chest X-ray on admission showed consolidation in the right lower lobe with an associated pleural effusion (figure 1). An electrocardiogram showed atrial fibrillation.

A chest X-ray performed after treatment showed radiographic resolution of the pneumonia. He re-presented 4 months later with a further right lower lobe pneumonia. A computed tomography (CT) scan of the thorax was performed (figure 2) and bronchoscopy was carried out.
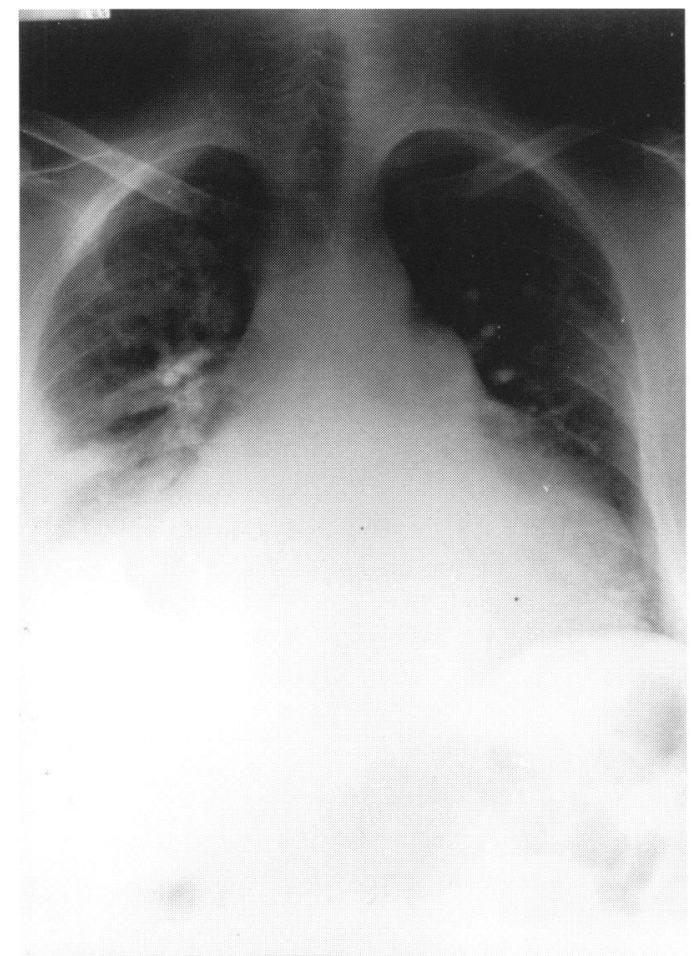

\footnotetext{
Department of Medicine, Glan Clwyd Hospital, Rhyl,

Denbighshire LL18 5UJ, UK

N A McAndrew

G R Ellis

G J Green
}

Accepted 16 December 1997
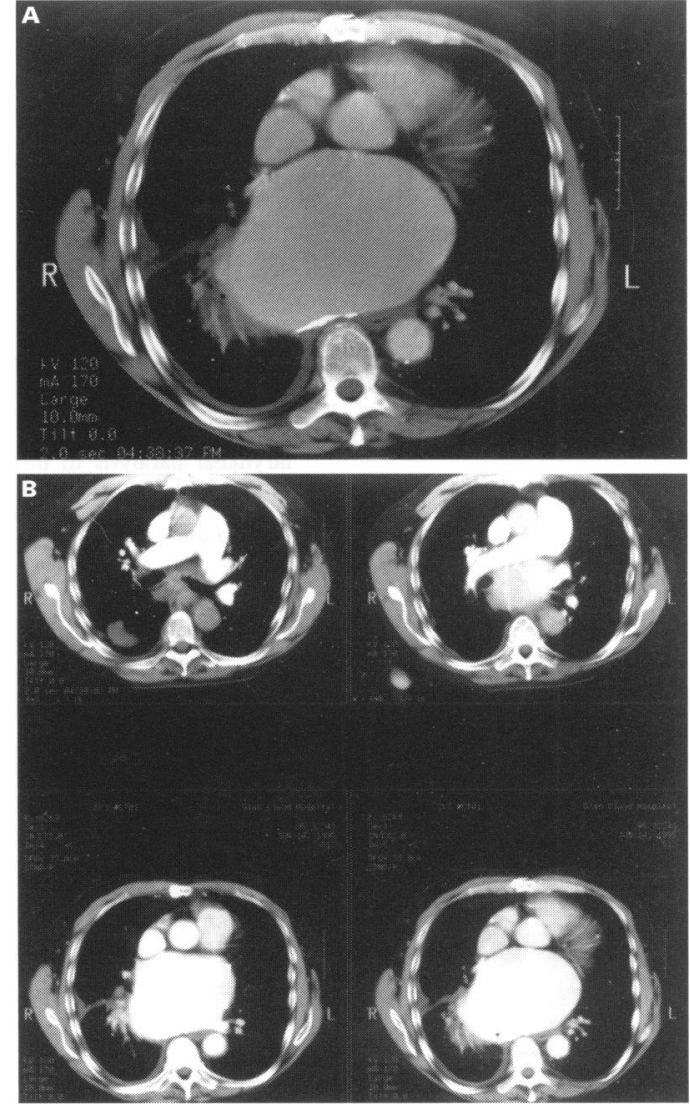

Figure 2 CT images of thorax

Figure 1 Chest X-ray, posterio-anterior view

\section{Questions}

1 What does the CT scan demonstrate and how is it related to this patient's recurrent pneumonia?

2 Name two other recognised non-cardiac complications of this condition?

3 What are the treatment options? 


\section{Answers}

QUESTION 1

The CT scan shows massive enlargement of the left atrium, the wall of which is calcified (figure 2A). The left atrium is compressing the right lower lobe bronchus (figure 2B). Bronchoscopy revealed stenosis of the right lower lobe bronchus caused by external compression.

QUESTION 2

Hoarseness and dysphagia.

QUESTION 3

Continued conservative management, bronchial stenting, and surgical correction.

\section{Discussion}

Massive enlargement of the left atrium can occur in pure mitral stenosis but is usually seen with severe, chronic mitral regurgitation. This patient initially had pure mitral stenosis but developed para-prosthetic mitral regurgitation after his second valve replacement. Recognised complications of massive left atrial enlargement include dysphagia due to compression of the oesophagus ${ }^{1}$ and hoarseness due to palsy of the left recurrent laryngeal nerve (Ortner's syndrome). The latter was originally thought to be due to compression of the nerve by an enlarged left atrium but is now accepted as being due to pulmonary artery dilatation. ${ }^{2}$

1 DeSanctus RW, Dean DC, Bland EF. Extreme left atrial enlargement, some characteristic features. Circulation 1964;29:14-23.

2 Stocker HH, Enterline HT. "Cardio-vocal syndrome": laryngeal paralysis in intrinsic heart disease. Am Heart $\dot{f}$

3 Bach F, Keith TS. Enlargement of the left auricle of the heart. Lancet 1929;2:766-7.

\section{Learning point}

Bronchial compression is a largely forgotten complication of massive left atrium which should be considered when recurrent pneumonia occurs in patients with mitral valve disease

Compression of the bronchi, especially the left, by a massive left atrium has been described for over 65 years $^{3}$ but is rarely mentioned in modern textbooks. Furthermore, it is well known that recurrent chest infections can occur in mitral valve disease but this is generally attributed to bronchial mucosal oedema caused by pulmonary artery hypertension.

Intrabronchial stents have been used in nonmalignant cases of bronchial stenosis ${ }^{4}$ but the potential long-term problems of inflammation and granuloma formation limit their use. Surgical treatment includes atrioplasty. ${ }^{5}$

\section{Final diagnosis}

Recurrent lobar pneumonia as a result of massive left atrium causing right lower lobe bronchial stenosis.

Keywords: mitral valve disease; bronchial stenosis; lobar pneumonia

4 Fox RA, Stanbridge RDeL, Brown J, Riordan JF, Rudd RM. Expandable metal stents for non-malignant bronchial obstruction. Thorax 1996;51:963-4.

5 Serra AJ, McNicholas KW, Lemole GM. Giant left atrium as cause of left pulmonary artery obstruction. Ann Thor Surg 1987;43:329-31. 